DALAT UNIVERSITY JOURNAL OF SCIENCE Volume 10, Issue 2, 2020 94-104

\title{
DENSITY OF CONDENSATE OF WEAKLY INTERACTING BOSE GAS CONFINED BETWEEN TWO HARD WALLS IN IMPROVED HARTREE-FOCK APPROXIMATION
}

\author{
Nguyen Van Thu ${ }^{\mathrm{a}^{*}}$ \\ ${ }^{a}$ The Faculty of Physics, Hanoi Pedagogical University 2, Hanoi, Vietnam \\ *Corresponding author: Email: nvthu@live.com \\ Article history \\ Received: July $27^{\text {th }}, 2019$ \\ Received in revised form: August $16^{\text {th }}, 2019 \mid$ Accepted: October $9^{\text {th }}, 2019$
}

\begin{abstract}
By means of the Cornwall-Jackiw-Tomboulis effective potential, the condensate density of a weakly interacting Bose gas confined between two hard walls is investigated within an improved Hartree-Fock approximation (IHF). Our results show that the condensate density in an IHF approximation is always bigger than the one in a double-bubble approximation and that the condensate density strongly depends on the distance between two walls as well as the gas parameter.
\end{abstract}

Keywords: Bose gas; Density of condensate; Finite-size effect; Improved Hartree-Fock approximation.

DOI: http://dx.doi.org/10.37569/DalatUniversity.10.2.579(2020)

Article type: (peer-reviewed) Full-length research article

Copyright (C) 2020 The author(s).

Licensing: This article is licensed under a CC BY-NC 4.0 


\title{
MậT ĐỘ NGƯNG TỤ CỦA KHÍ BOSE TƯƠNG TÁC YẾU B!̣ GIAM GIŨ๋A HAI TƯỜNG CÚNG TRONG GẦN ĐÚNG HARTREE-FOCK CẢI TIẾN
}

\author{
Nguyễn Văn Thụ ${ }^{a^{*}}$ \\ ${ }^{a}$ Khoa Vật lý, Truò̀ng Đại học Su phạm Hà Nội 2, Hà Nội, Việt Nam \\ "Tác giả liên hệ: Email: nvthu@live.com \\ Lịch sử bài báo \\ Nhận ngày 27 tháng 7 năm 2019
}

Chỉnh sửa ngày 16 tháng 8 năm 2019 | Chấp nhận đăng ngày 09 tháng 10 năm 2019

\section{Tóm tắt}

Bằng cách sủ dụng thế hiệu dụng Cornwall-Jackiw-Tomboulis, chúng tôi nghiên cưu mật độ ngung tu của khi Bose tuơng tác yếu bị giam giũ giữa hai tuờng cưng trong gần đúng Hartree-Fock cải tiến (IHF). Các kết quả của chúng tôi chỉ ra rằng mật độ ngưng tu trong gần đúng IHF luôn lớn hơn giá trị của nó trong gần đúng hai vòng và nó phụ thuộc mạnh vào khoảng cách giữa hai tuờng cứng cũng nhu thông số khí.

Từ khóa: Gần đúng Hartree-Fock cải tiến; Hiệu ứng kích thước hữu hạn; Khí Bose; Mật độ ngưng tụ.

DOI: http://dx.doi.org/10.37569/DalatUniversity.10.2.579(2020)

Loại bài báo: Bài báo nghiên cứu gốc có bình duyệt

Bản quyền @ 2020 (Các) Tác giả.

Cấp phép: Bài báo này được cấp phép theo CC BY-NC 4.0 


\section{INTRODUCTION}

Although it was predicted in 1925, studies on Bose gas are, and will be, a current problem in modern physics. There are numerous papers in this field, including ones on the ground state (Ao \& Chui, 1998; Barankov, 2002), surface tension and Antonov wetting line (Indekeu, Lin, Nguyen, Schaeybroeck, \& Tran, 2015; Nguyen \& Hoang, 2018), dynamics of surface excitation (Indekeu, Nguyen, Lin, \& Tran, 2018; Pethick \& Smith, 2008) and so on.

The finite-size effect on a Bose gas, which appears when one or more dimensions of the space are reduced, is one of the most interesting problems in this field, and one that has attracted the attention of many physicists. The forces on a single Bose gas confined between two parallel plates were calculated by Nguyen (2018). The influence of the finite-size effect on the order parameter and Casimir force was considered in the improved Hartree-Fock approximation (Nguyen \& Luong, 2018). The finite-size effect was also investigated for two-component Bose-Einstein condensates (Nguyen \& Luong, 2017; Nguyen \& Luong, 2019). However, these studies only concentrate on the Casimir effect and surface tension. To our knowledge, the study of the condensate density of a Bose gas under a finite-size effect constraint is still absent.

In studying properties of a Bose gas, the condensate density plays an important role and, based on it, we are able to calculate every thermodynamic quantity. Up to now two methods are usually employed to consider the condensate density of a Bose gas, namely, Gross-Pitaevskii (GP) theory and quantum field theory in the formalism of the Cornwall-Jackiw-Tomboulis (CJT) effective potential. However, in the GP theory, the quantum fluctuations are neglected (Pethick \& Smith, 2008), whereas these fluctuations are taken into account in the CJT effective potential (Andersen, 2004) with several levels of approximation, such as the one-loop, double-bubble, and improved HartreeFock approximation. In this paper we consider the effect of the compaction in one direction on the density of state by means of the CJT effective potential. In order to obtain highly accurate results, the improved Hartree-Fock approximation (IHF), in which the number of Goldstone bosons is conserved, is invoked (Ivanov, Riek, \& Knoll, 2005).

\section{EQUATIONS OF STATE IN THE HARTREE-FOCK APPROXIMATION}

To begin with, we consider a system of dilute Bose gas described by the Lagrangian (Pethick \& Smith, 2008) in Equation (1):

$$
L=\psi *\left(-i \hbar \frac{\partial}{\partial t}-\frac{\hbar^{2}}{2 m} \nabla^{2}\right) \psi-\mu \psi^{2}+\frac{g}{2} \psi^{4},
$$

Which $\psi=\psi(\vec{r}, t)$ is the field operator; $m$ and $\mu$ are the atomic mass and chemical potential, respectively; $\hbar$ is Plack's constant and coupling constant in Equation (2): 


$$
g=4 \pi \hbar^{2} a_{s} / m>0
$$

determines the strength of repulsive intraspecies interactions via the scattering length $a_{s}$ of the $s$-wave. Note that we neglect particle flow and external potential so that the field operator is real.

We now establish the equations of state, which govern all changes in state of the system. In order to do that, the field operator should be shifted (Andersen, 2004) in Equation (3):

$$
\psi \rightarrow \psi_{0}+\frac{1}{\sqrt{2}}\left(\psi_{1}+i \psi_{2}\right)
$$

Where $\psi_{0}$ is the expectation value of the field operator in the treeapproximation, which plays the role of order parameter, and $\psi_{1}, \psi_{2}$ are the quantum fluctuations of the field. Putting (3) into Lagrangian (1) form one has the interacting Lagrangian in the double-bubble approximation (Nguyen \& Luong, 2018) in Equation (4):

$$
L_{\mathrm{int}}=\frac{g}{2} \psi_{0} \psi_{1}\left(\psi_{1}^{2}+\psi_{2}^{2}\right)+\frac{g}{8}\left(\psi_{1}^{2}+\psi_{2}^{2}\right)^{2} .
$$

The Cornwall-Jackiw-Tomboulis (CJT) effective potential can be read off from (4):

$$
V_{\beta}^{C J T}=-\mu \psi_{0}^{2}+\frac{g}{2} \psi_{0}^{4}+\frac{1}{2} \int_{\beta} \operatorname{Tr}\left[\ln D^{-1}(k)+D_{0}^{-1}(k) D(k)-I\right]+\frac{3 g}{8}\left(P_{11}^{2}+P_{22}^{2}\right)+\frac{g}{4} P_{11} P_{22},
$$

With $I$ being a unit matrix, $\vec{k}$ wave vector, notation $\beta=1 / k_{B} T$ with Boltzmann constant $k_{B}$ and temperature $T$. In Equation $(5), D(k)$ is the propagator in the doublebubble approximation, which is reduced, (5) and (6) is the inversion propagator in the tree-approximation.

$$
D_{0}^{-1}(k)=\left(\begin{array}{cc}
\frac{\hbar^{2} k^{2}}{2 m}+2 g \psi_{0}^{2} & -\omega_{n} \\
\omega_{n} & \frac{\hbar^{2} k^{2}}{2 m}
\end{array}\right),
$$

The Matsubara frequency for bosons is defined as $\omega_{n}=2 \pi n / \beta, n=0,1,2 \ldots$ In Equation (5), we also use the notation: $\int_{\beta} f(k)=\frac{1}{\beta} \sum_{n=-\infty}^{\infty} \frac{d^{3} \vec{k}}{(2 \pi)^{3}} f\left(\omega_{n}, \vec{k}\right)$.

By requiring the determinant to (6) vanish, the dispersion relation has the form (7): 


$$
E(k)=\sqrt{\frac{\hbar^{2} k^{2}}{2 m}\left(\frac{\hbar^{2} k^{2}}{2 m}+2 g \psi_{0}^{2}\right)} .
$$

Equation (7) shows that in the tree approximation there is a Goldstone boson associated with $U(1)$ breaking. However, the CJT effective potential will not give any Goldstone boson (Tran, Le, Nguyen, \& Nguyen, 2009). To restore this boson, the method proposed by Ivanov et al. (2005) is employed by adding a term into the CJT effective potential (Nguyen \& Luong, 2018) (Equation 8).

$$
\Delta V=-\frac{g}{4}\left(P_{11}^{2}+P_{22}^{2}-2 P_{11} P_{22}\right)
$$

Combining Equations (5) and (8) one gets a new CJT effective potential (Equation 9).

$$
\begin{aligned}
\tilde{V}_{\beta}^{C J T}=V_{\beta}^{C J T}+\Delta V= & -\mu \psi_{0}^{2}+\frac{g}{2} \psi_{0}^{4}+\frac{1}{2} \int_{\beta} \operatorname{Tr}\left[\ln D^{-1}(k)+D_{0}^{-1}(k) D(k)-I\right] \\
& +\frac{g}{8}\left(P_{11}^{2}+P_{22}^{2}\right)+\frac{3 g}{4} P_{11} P_{22} .
\end{aligned}
$$

It is easy to verify that the $C J T$ effective potential (9) reproduces the Goldstone boson with a new dispersion relation (Tran et al., 2009) in Equation (10):

$$
E(k)=\sqrt{\frac{\hbar^{2} k^{2}}{2 m}\left(\frac{\hbar^{2} k^{2}}{2 m}+M\right)},
$$

With $M$ being the effective mass. This is the reason why this approximation is called the IHF approximation. Minimizing Equation (9) with respect to the order parameter and elements of the propagator one arrives at the gap Equation (11).

$$
-\mu+g \psi_{0}^{2}+\frac{3 g}{2} P_{11}+\frac{g}{2} P_{22}=0
$$

and the Schwinger-Dyson (SD) Equation (12):

$$
M=-\mu+3 g \psi_{0}^{2}+\frac{g}{2} P_{11}+\frac{3 g}{3} P_{22}
$$

Equation (11) and (12) are called equations of state, which allow us to calculate the effective mass $M$, and especially the order parameter $\psi_{0}$ and therefore the condensate density in Equation (13):

$$
\rho_{0}=\psi_{0}^{2}
$$


In order to proceed further, one has to work with momentum integrals $P_{11}$ and $P_{22}$. At zero temperature, these integrals become (Nguyen \& Luong, 2019) in Equation (14):

$$
P_{11}=\frac{1}{2} \int \frac{d^{3} \vec{k}}{(2 \pi)^{2}}\left(\frac{\hbar^{2} k^{2} / 2 m}{\hbar^{2} k^{2} / 2 m+M}\right), P_{22}=\frac{1}{2} \int \frac{d^{3} \vec{k}}{(2 \pi)^{2}}\left(\frac{\hbar^{2} k^{2} / 2 m+M}{\hbar^{2} k^{2} / 2 m}\right) .
$$

\section{DENSITY OF CONDENSATE OF WEAKLY INTERACTING BOSE GAS CONFINED BETWEEN TWO HARD WALLS}

Consider a weakly interacting Bose gas confined between two hard walls. These walls are perpendicular to the $0 z$ axis and separated at distance $\ell$, along the $0 x, 0 y$ directions, the system under consideration is translational. Because of the compaction in the z-direction, the wave vector is quantized in Equation (15):

$$
k^{2} \rightarrow k_{\perp}^{2}+k_{j}^{2}, j=1,2,3 \ldots
$$

Which $k_{\perp}$ and $k_{j}$ are perpendicular to and parallel with $0 z$. For a boson system, the periodic boundary condition is employed at the hard walls (Nguyen, 2018) in Equation (16):

$$
k_{j}=\frac{2 \pi j}{\ell}, j \in \mathbf{Z}
$$

For simplicity, one now converts all relevant quantities into dimensionless form by introducing the healing length $\xi=\hbar / \sqrt{2 m g n_{0}}$ with $n_{0}$ being the bulk density. The dimensionless length is $L=\ell / \xi, \tau=z / \xi$ and the dimensionless wave vector is $\kappa=k \xi$. Equation (15) can be rewritten as $\kappa^{2} \rightarrow \kappa_{\perp}^{2}+\kappa_{j}^{2}$ and the momentum integrals (14) become Equation (17):

$$
P_{11}=\frac{1}{4 \pi \xi^{3}} \int_{0}^{\Lambda} \kappa_{\perp} d \kappa_{\perp} \sum_{j=-\infty}^{\infty}\left(\frac{\kappa_{\perp}^{2}+\kappa_{j}^{2}}{\kappa_{\perp}^{2}+\kappa_{j}^{2}+\mathrm{M}^{2}}\right), P_{22}=\frac{1}{4 \pi \xi^{3}} \int_{0}^{\Lambda} \kappa_{\perp} d \kappa_{\perp} \sum_{j=-\infty}^{\infty}\left(\frac{\kappa_{\perp}^{2}+\kappa_{j}^{2}+\mathrm{M}^{2}}{\kappa_{\perp}^{2}+\kappa_{j}^{2}}\right)
$$

Where $\mathrm{M}=M / g n_{0}$ and $\Lambda$ is a momentum cut-off, which is introduced to avoid the UV-divergence in integrating over $\kappa_{n}$. The summation in Equation (17) can be dealt with the aid of the Euler-Maclaurin formula (Arfken \& Weber, 2005) and then by taking $\Lambda \rightarrow \infty$ one obtains in Equation (18):

$$
P_{11}=0, P_{22}=\frac{m g n_{0}}{12 \hbar^{2} \xi L} \mathrm{M}^{1 / 2}
$$


We now move to study the density of condensate. To do this, equations of state should be reduced to dimensionless form by using $\phi_{0}=\psi_{0} / \sqrt{n_{0}}$ keeping in mind that the system under consideration is connected to a particle reservoir, which associates with a grand canonical ensemble. Substituting Equation (18) and (2) into Equation (11) and (12) one has the equations of state in dimensionless form in Equation (19):

$$
-1+\phi_{0}^{2}+\frac{\sqrt{2 \pi^{3}} n_{s}^{1 / 2} \mathrm{M}^{1 / 2}}{3 L}=0, \mathrm{M}=-1+3 \phi_{0}^{2}+\frac{\sqrt{2 \pi^{3}} n_{s}^{1 / 2} \mathrm{M}^{1 / 2}}{L},
$$

Which $n_{s}=n_{0} a_{s}^{3} \ll 1$ because of the condition for a dilute Bose gas, and it is called the gas parameter (Pethick \& Smith, 2008). The solution for Equation (19) can be easily found by Equation (20):

$$
\phi_{0}^{2}=1+\frac{2 \pi^{3 / 2} n_{s}^{1 / 2}}{3 L}, \mathrm{M}=2
$$

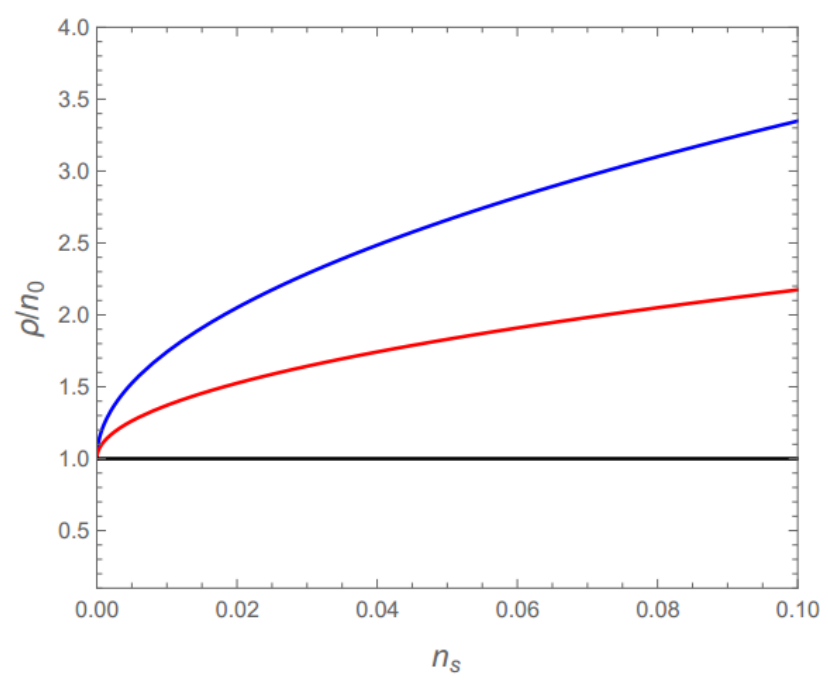

Figure 1. Density of condensate versus the distance $L$

Note: The red and blue lines correspond to $\rho_{0} / n_{0}$ and $\rho_{I H F} / n_{0}$.

Combining (20) and (13) one finds the density of condensate (Equation 21):

$$
\rho_{0}=n_{0}\left(1+\frac{2 \pi^{3 / 2} n_{s}^{1 / 2}}{3 L}\right)
$$

As an illustration for the above calculations, numerical computations are made for rubidium $\mathrm{Rb} 87$ (Egorov et al., 2013) with $m=86.9 \mathrm{u}, a_{s}=50 \AA \mathrm{A}, \xi=4000 \AA$. . The result for $\rho_{0} / n_{0}$ versus dimensionless distance is shown in Figure 1 by the red line. Let us now investigate the condensate density starting from the CJT effective potential. To this end, one begins with the definition of the pressure (Equation 22): 


$$
P=-\left.\tilde{V}_{\beta}^{C J T}\right|_{\text {at minimum }}
$$

Which the subscript "at minimum" means that the CJT effective potential is taken providing that it consists with (11) and (12). The density of condensate in the IHF approximation is now defined by Equation (23):

$$
\rho_{I H F}=\frac{\partial P}{\partial \mu} .
$$

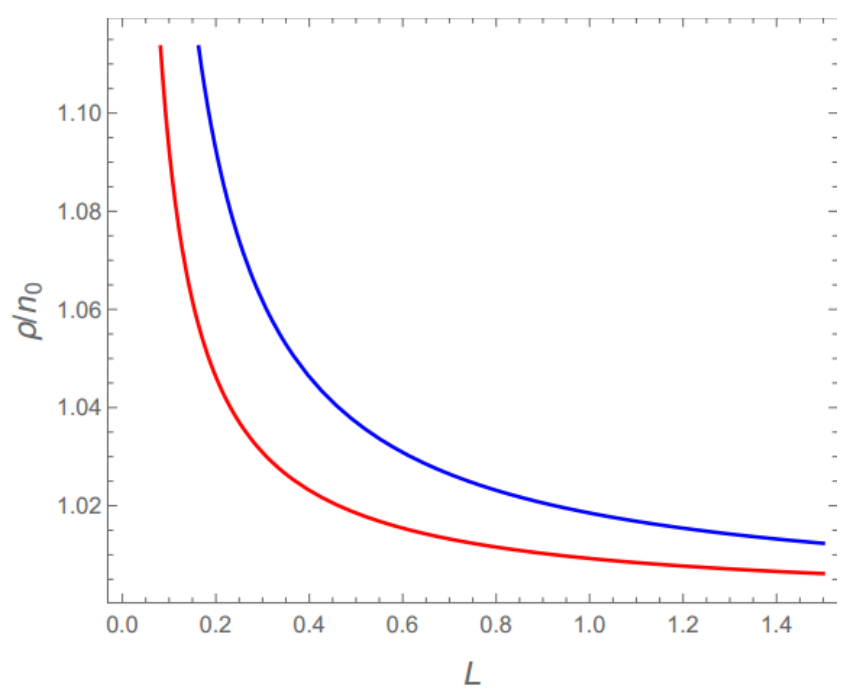

Figure 2. The $n_{s}$ - dependence of density of condensate

Note: The red and blue lines correspond to $\rho_{0} / n_{0}$ and $\rho_{I H F} / n_{0}$.

Plugging (9) into (23) leads to Equation (24):

$$
\rho_{I H F}=\psi_{0}^{2}+\frac{1}{2}\left(P_{11}+P_{22}\right)
$$

From (18), (20), and (24), the density of condensate in the IHF approximation becomes Equation (25):

$$
\rho_{I H F}=n_{0}\left(1+\frac{4 \pi^{3 / 2} n_{s}^{1 / 2}}{3 L}\right) .
$$

The blue line in Figure 1 graphically shows the evolution of $\rho_{I H F}$ as a function of dimensionless distance $L$ with the same parameters as for the red line. It is clear that both $\rho_{0}$ and $\rho_{I H F}$ are divergent when the distance approaches zero and decay rapidly when the distance increases. Both tend to $n_{0}$ at large $L$, at which the influence of the finite-size effect can be ignored. At a given value of the distance between two hard walls, the density of condensate in the IHF approximation is always the same in both 
mean field theory and one-loop approximation. This fact is explained due to the contribution of the last term in the right-hand side of (24) and (25). Physically, it is the contribution of the high-order term in the interacting Lagrangian (4) so that this is a more exact result for the density of condensate in comparison with the others.

Figure 2 shows the evolution of $\rho_{0}$ (red line) and $\rho_{I H F}$ (blue line) as functions of the gas parameter $n_{s}$ with the same parameters as in Figure 1, the black line corresponds to the condensate density in the one-loop approximation. For each value of the gas parameter, the $\rho_{I H F}$ is larger than the condensate density in both the tree-approximation and the one-loop approximation because of the contribution from high-order diagrams. Note that our system is connected to the particle reservoir so that the density of condensate increases as the gas parameter increases, which is a consequence of increasing the bulk density $n_{0}$. An important result is that at $n_{s}=0$ one has $\rho_{0}=\rho_{I H F}=n_{0}$. This means that, for an ideal Bose gas, the one-loop approximation is accurate enough to study the condensate density.

\section{CONCLUSIONS AND DISCUSSIONS}

In the foregoing sections, using mean field theory in the formalism of CornwallJackiw-Tomboulis effective potential with the improved Hartree-Fock approximation, which conserves the number of Goldstone bosons, we studied the density of condensate of a dilute Bose gas confined between two plates. Our main results are, in order:

- Because of the very small gas parameter, analytical relations for $\rho_{0}$ and $\rho_{I H F}$ are attained. Their values are equal to those in the tree approximation after adding an extra term, which depends on the distance between two hard walls and the gas parameter;

- Our analytical solutions and numerical computations show that when the gas parameter is fixed and at a given value of the distance $L, \rho_{I H F}$ is always greater than $\rho_{0}$. The difference is explained due to the contribution of high order diagrams in the IHF approximation;

- By considering the effect from the gas parameter we proved that for an ideal Bose gas, the one-loop approximation is adequate for considering the density of condensate.

Based on this result, it is possible to study the influence of the finite-size effect on the pressure of a Bose gas as well as the density of condensate of a binary mixture of Bose gases. 


\section{ACKNOWLEDGEMENT}

This research is funded by the Vietnam National Foundation for Science and Technology Development (NAFOSTED) under grant number 103.01-2018.02.

\section{REFERENCES}

Andersen, J. O. (2004). Theory of the weakly interacting Bose gas. Rev. Mod. Phys. 76, 599-639.

Ao, P., \& Chui, S. T. (1998). Binary Bose-Einstein condensate mixtures in weakly and strongly segregated phases. Physical Review A, 58(6), 4836-4840.

Arfken, G. B., \& Weber, H. J. (2005). Mathematical Methods for Physicists. California, USA: San Diego Academic.

Barankov, R. A. (2002). Boundary of two mixed Bose-Einstein condensates. Physical Review A, 66, 1-5.

Egorov, M., Opanchuk, B., Drummond, P., Hall, B. V., Hannaford, P., \& Sidorov, A. I. (2013). Measurement of s-wave scattering lengths in a two-component BoseEinstein condensate. Physical Review A, 87, 3614-3620.

Indekeu, J. O., Lin, C. Y., Nguyen, V. T., Schaeybroeck, B. V., \& Tran, H. P. (2015). Static interfacial properties of Bose-Einstein-condensate mixtures. Physical Review A, 91, 3615-3621.

Indekeu, J. O., Nguyen, V. T., Lin, C. Y., \& Tran, H. P. (2018). Capillary-wave dynamics and interface structure modulation in binary. Physical Review A, 97, 3605-3612.

Ivanov, Y. B., Riek, F., \& Knoll, J. (2005). Gapless Hartree-Fock resummation scheme for the $\mathrm{O}(\mathrm{N})$ model. Physical Review D, 71, 5016-5023.

Nguyen, V. T. (2018). The forces on a single interacting Bose-Einstein condensate. Phys. Lett. A, 382, 1078-1085.

Nguyen, V. T., \& Hoang, V. Q. (2018). Antonov wetting line phase transition of twocomponent Bose-Einstein condensates under constraint of Robin boundary condition. Dalat University Journal of Science, 8(3), 61-68.

Nguyen, V. T., \& Luong, T. T. (2017). Casimir force of two-component Bose-Einstein condensates confined by a parallel plate geometry. Journal of Statistical Physics 168(1), 1-10.

Nguyen, V. T., \& Luong, T. T. (2018). Influence of the finite size effect on properties of a weakly interacting Bose gas in improved Hartree-Fock approximation. Vietnam National University Journal of Science, 34(3), 43-47.

Nguyen, V. T., \& Luong, T. T. (2019). Finite-size effect on Bose Einstein condensate mixtures in improved Hartree-Fock approximation. Int. J. Mod. Phys. B, 33(12), $1-10$. 
Pethick, C. J., \& Smith, H. (2008). Bose-Einstein condensation in dilute gas. Cambridge, UK: Cambridge University Press.

Tran, H. P., Le, V. H., Nguyen, T. A., \& Nguyen, V. L. (2009). Bose-Einstein condensation in binary mixture of Bose gases. Ann. Phys. 324, 2074-2085. 